\title{
Humanized mouse models to study cell-mediated immune responses to liver stage malaria vaccines
}

\author{
Michael F. Good ${ }^{1,2}$, Michael T. Hawkes ${ }^{2,3,4}$, Stephanie K. Yanow ${ }^{2,4,5}$ \\ ${ }^{1}$ Institute for Glycomics, Griffith University, Australia \\ ${ }^{2}$ Department of Medical Microbiology and Immunology, University of Alberta, \\ Edmonton, AB, Canada \\ ${ }^{3}$ Department of Pediatrics, Division of Infectious Diseases, University of Alberta, \\ Edmonton, AB, Canada \\ ${ }^{4}$ School of Public Health, University of Alberta, Edmonton, AB, Canada \\ ${ }^{5}$ Provincial Laboratory for Public Health, Edmonton, AB, Canada \\ Corresponding author: Good, M. F. (michael.good@griffith.edu.au) \\ Keywords: malaria, vaccines, humanized mice, liver-stage immunity.
}




\section{Abstract}

Malaria vaccine development is hampered by the lack of small animal models that recapitulate human immune responses to Plasmodium falciparum. We review the burgeoning literature on humanized mice for $P$. falciparum infection, including challenges in engraftment of human immune cells, hepatocytes, and erythrocytes. Recent advances in immune-compromised mouse models and stem cell technology have already enabled proof-of-concept that the entire parasite life cycle can be sustained in a murine model and that adaptive human immune responses to several parasite stages can be measured. Nonetheless, optimization is needed to achieve a reproducible and relevant murine model for malaria vaccine development. This review is focused on the complexities of T cell development in a mouse humanized with both a lymphoid system and hepatocytes. An understanding of this will facilitate the utility of humanized mice in the development of liver stage vaccines.

\section{Malaria vaccine approaches}

Malaria is a major pathogen that is responsible for nearly one million deaths annually, most of which occur in young children in sub-Saharan Africa [1]. The disease is caused by an intracellular parasite (genus Plasmodium) that infects human hepatocytes and red blood cells, and is transmitted by mosquitoes. In the human host, sporozoites exist outside of a human cell; these are inoculated by the mosquito and enter a hepatocyte where they develop over a period of 6-7 days into thousands of merozoites through a process of schizogony (Figure 1). These rupture from the liver cell and invade a red blood cell where, for $P$. falciparum, there is a $48 \mathrm{~h}$ developmental cycle. A single 
merozoite gives rise to a schizont that contains 16-30 individual nuclei forming merozoites. Rupture of the red blood cell releases merozoites that then invade new red cells. Thus, there is exponential growth of the parasite within the red cell compartment. Six different species of Plasmodium are known to infect humans; $P$. falciparum is the most deadly.

More than 40 candidate malaria vaccines have reached the clinical trial stage [2]. Vaccine strategies may target the pre-erythrocytic stage, blood stage, or sexual stages (transmission-blocking). An advantage of pre-erythrocytic vaccines is the potential to completely block infection, preventing parasites from reaching the blood and causing disease, as well as onward transmission. Current pre-erythrocytic vaccines exploit a variety of approaches. (i) Genetically engineered surface antigens from the sporozoite or infected hepatocyte. The RTS,S vaccine is the most advanced in the vaccine development pipeline but has a protective efficacy of only $27-46 \%$ in infants and children [3]. (ii) DNA and viral vectored delivery to induce a cellular immune response [4, 5]. (iii) Live, attenuated whole sporozoites. The most advanced whole parasite vaccine, known as PfSPZ, consists of the entire sporozoite, dissected from mosquitoes, irradiated and vialed [6]. A recently published human study in a small number of volunteers showed that it induced complete protection from challenge [7]. When tested in Rhesus monkeys, this vaccine induced an immune response in the liver that involves T cells [8]. The findings from the viral-vectored and whole sporozoite vaccine studies support an important role for CD8+ T-lymphocyte responses in pre-erythrocytic vaccine efficacy. Pre-clinical development of the next generation of anti-malaria vaccines will benefit from models that recapitulate cell- 
mediated immunity to liver-stage parasites (see below) and the development of such models will be the subject of this review.

\section{Immunity to malaria in the liver}

Our understanding of malaria immunity to liver stage parasites largely stems from studies with irradiated sporozoites. Ruth Nussenzweig and colleagues first demonstrated that irradiated sporozoites from a rodent malaria species could induce immunity to subsequent sporozoite challenge [9]. In this and subsequent studies, immunity was shown to be stage-specific (mice were not protected against challenge with blood stage parasites), species-specific, and associated with the production of antibodies to the circumsporozoite (coat) protein of the sporozoite. Sporozoites were initially delivered by the bite of irradiated mosquitoes. Subsequent studies were undertaken in human volunteers immunized by the bite of irradiated mosquitoes (e.g. [10]) where immunity was also observed. More recently, human volunteers immunized with large numbers of purified irradiated $P$. falciparum sporozoites were protected from subsequent challenge infection with sporozoites [7].

T cell depletion studies in mice demonstrated that CD8+ T cells were critical for immunity $[11,12]$ and studies in Rhesus monkeys immunized with $P$. falciparum sporozoites showed that interferon- $\gamma$-producing parasite-specific CD8+ T cells were found in the livers of immunized animals [8]. There has been debate as to where CD8+ T cells are primed following immunization with irradiated sporozoites, with evidence supporting priming in the draining lymph nodes of the skin and other data suggesting that infected hepatocytes can directly prime T cells (reviewed in [13]. 
The mechanisms by which CD8+ T cells are then able to eliminate infected hepatocytes are not well understood, but it appears likely that cytokines (IFN- $\gamma$, TNF$\alpha)$ are involved. Differences in critical immune responses may occur between different individuals and between different species of Plasmodium [14]. Live imaging of the liver during killing of infected hepatocytes by $\mathrm{T}$ cells shows that $\mathrm{T}$ cells recruit other T cells to an infected hepatocyte and that a large cluster of T cells is involved in killing the infected cell over a prolonged period of time (gang killing) [15].

These studies in human volunteers are extremely expensive and laborious. It is not possible to study in detail the immune responses occurring in the livers of humans immunized with irradiated sporozoites or with other malaria vaccines. Chimpanzees and other primates are not easily available for both ethical and financial reasons. Mice are widely used in research but human Plasmodium species do not infect mice, leaving researchers with no choice but to use analogous rodent species. Extrapolations from studies where mice are immunized with rodent parasites or vaccines to protect against rodent parasites are not always reliable. The purpose of this review is to describe the strategies behind the design of various humanized mouse models, along with their limitations, as they may apply to the development of malaria vaccines that target the human parasite during the liver stage of its life cycle.

\section{The need for humanized leukocyte-liver (HLL) mice}

'Humanized' mice are a revolutionary research tool for the pre-clinical study of human infectious diseases. Through genetic manipulation, selective breeding, and various approaches to avoid graft rejection, mice with high levels of human 
chimerism can be routinely generated. Upon transplantation with specific human tissues or cells (e.g. hepatocytes), these mice provide a localized human environment that supports infection by human pathogens. Engraftment of human immune cells could then permit the study of the host response to infection, thereby making these animals ideally suited for pre-clinical evaluation of vaccines [16, 17].

For malaria, humanized leukocyte-liver (HLL) mice could be immunized with experimental vaccines to stimulate an immune response and challenged with human malaria infection to assess protection against liver stage parasites. Currently the only ways to demonstrate liver stage immunity to a malaria vaccine are to either perform a liver resection or use luminescent parasites and measure a reduction in parasite RNA or protein expressed during the liver stage (e.g. [18]. While this cannot be done in human studies, it is a straightforward procedure in mice.

Once an experimental vaccine is shown to induce protection against human malaria in the HLL mouse, this model could further serve as a valuable tool to optimize the dosage and delivery of the vaccine in preparation for human trials. Pre-clinical optimization in the HLL mouse could become an integral component in selecting vaccines to advance to clinical trials. Tremendous cost savings would be achieved and the chances of success in human trials would be more favourable. Furthermore, the possibility of testing combinations of vaccines with different targets is also feasible. Another significant advantage is the ability to challenge the mice with both homologous and heterologous parasite strains and species (eg. P. vivax). Mice could be challenged by mosquito bite, injected sporozoites, or blood stage parasites to evaluate the immune response to these different exposures to malaria. Feeding of 
mosquitoes on the vaccinated mice could also assess transmission-blocking activity of a vaccine. Collectively, the HLL mouse is a powerful tool to support the transition of experimental vaccines from discovery research to clinical trials.

The HLL mouse is particularly relevant for studying vaccines that target the liver stage of malaria. These include: irradiated, chemically attenuated, or genetically attenuated whole sporozoite vaccines [7, 19, 20]; DNA prime boost and viral vectored subunit vaccines [4]; the vaccine approach whereby liver stage immunity is induced following exposure to low numbers of sporozoites with chloroquine chemoprophylaxis (CPS) [21]; and possibly other vaccines that induce CD8+ T cell responses (e.g. chemically attenuated blood stage parasites [22]).

\section{Developing HLL mice for vaccine research}

While mice with a humanized immune system have been under development for nearly 30 years [23], and mice with a humanized liver were developed over the last 15 years [24], attempts to construct mice with both a humanized immune system and liver have been undertaken only recently. Many challenges remain but this is an exciting vaccine research opportunity.

The first challenge is to identify or generate a suitable mouse strain that will permit the engraftment of human immune cells and hepatocytes. It is critical to ensure that the immune system of the mouse does not reject the human hepatocytes and the human lymphoid tissue. The general principles are to engineer mice that are deficient in innate and adaptive immune responses. The next challenge is to re-constitute the mouse with 
appropriate human immune cells, or their precursors. This will lead to a humanized immune system (HIS) mouse. Typically, stem cells (e.g. from the cord blood of a newborn baby) are used. These give rise to antigen presenting cells (such as dendritic cells) and to T cell progenitors. These progenitors must then mature in the mouse thymus gland to generate mature T cells. This is a critical challenge in HLL mouse construction. It is also necessary to ensure that the grafted human immune system does not cause graft-versus-host disease in the mouse. The third challenge is to engraft human hepatocytes. For this to occur, the mouse’s own hepatocytes must first be destroyed. This is achieved by activating specific transgenes in the mice designed to produce a liver toxin or by deleting genes required for hepatocyte survival. Donor human hepatocytes are then injected into the recipient, usually via the intra-splenic route.

Generating the basic humanized immune system mouse

There are now many different types of immune-deficient mice that can be used to accept human cells. Suitable mice have a phenotype of severe combined immune deficiency (deficient in their own T and B cells). In one commonly used humanized mouse model, recipient immune deficiency is conferred by a loss of function mutation in the Prkdc gene, encoding a DNA-dependent protein kinase catalytic subunit which functions in DNA non-homologous end-joining (commonly referred to as scid). Other commonly used strains have genetic mutations in the recombination activating genes, Rag1or Rag2, which encode enzymes that participate in immunoglobulin and T cell receptor VDJ recombination. These genetic alterations are usually referred to as ' $\mathrm{S}$ ' (SCID) or 'R' (Rag). However, SCID or Rag-/- mice are not fully suited to the generation of HLL mice without further genetic manipulations. They have natural 
killer (NK) cells and other innate immune responses that can limit human cell engraftments.

A genetic knockout of the interleukin 2 receptor gamma (IL2R $\gamma$ ) chain is commonly used to control NK activity and reduce the effects of inflammatory cytokines and chemokines. These mice are unable to signal via cytokines that require the IL2R $\gamma$ chain (IL2, IL4, IL7, IL9, IL15 and IL21) thereby eliminating NK cell activity and other inflammatory sequellae. This genetic alteration is usually referred to as 'G'. Mice with different genetic alterations in the $I L 2 R \gamma$ gene have been produced [16]). Because they are so severely immune-compromised, they have other defects in lymph node and B cell development even following immune reconstitution with human stem cells.

Breeding scid mice onto the non-obese diabetic (NOD) background [25] further generates a xenotransplantation-tolerant mouse with phagocytic dysfunction. This occurs because the recipient mouse $\operatorname{SIRP} \alpha$ gene closely resembles the human $\operatorname{SIRP} \alpha$ gene, thus reducing phagocytosis of xenogeneic cells (e.g. human stem cells) by macrophages via the CD47-SIRP $\alpha$ interaction. SIRP $\alpha$ provides a signal to macrophages not to remove self cells; thus if the SIRP $\alpha$ gene is similar to the human gene, removal of foreign cells by macrophages will be lessened [26]. The NOD background is usually referred to by the letter ' $N$ '.

These three basic genetic strategies come together to result in NSG and NRG mice. Another mouse is the NOG mouse which is similar to the NSG mouse but with a different $I L 2 R \gamma$ genetic alteration. While all these mice can readily accept human stem cells, there are still obstacles in the differentiation from stem cell to mature 
lymphocyte that must be overcome in order to use these mice for vaccine studies. Other related immunodeficient strains have been used and recently reviewed [27, 28].

Human CD34+ stem cells are a source of progenitors for T cells and antigen presenting cells that can be readily purified from newborn umbilical cord blood, adult blood or human fetal tissue, and are commercially available. Sufficient stem cells to generate 10-20 humanized mice can be readily obtained from one cord. The progenitors must then develop into T cells. This happens in the thymus gland through a process involving positive and negative selection to expand the repertoire of antigen-specific $\mathrm{T}$ cells while removing self-reactive T cells [29]. T cells are selected or 'educated' in the thymus to recognize foreign peptide epitopes presented by major histocompatibility complex (MHC) molecules expressed in the thymus. However, there are two significant issues in generating functional human $\mathrm{T}$ cells in a mouse that can recognize malaria parasite-infected human liver cells.

(i) The T cells are generated in a thymus where the MHC type of the thymus gland will play a major role in selecting the repertoire of antigen-specific T cells that emerge. Cells that develop in a gland that expresses solely mouse MHC antigens will have a repertoire that is skewed to recognize foreign antigenic peptides in association with mouse MHC molecules. Some will recognize peptides in association with human MHC molecules but these will be a minority. As discussed above, CD8+ T cells are the principal effector cells in malaria liver-stage immunity and these recognize antigenic peptides with MHC class I antigens. For efficient expansion and recognition of infected human liver cells, the thymus in which the $\mathrm{T}$ cells are educated should ideally express the same MHC class I antigens that are expressed in the hepatocytes. 
(ii) For expansion into effector and memory cells, CD8+ T cells will require antigenprimed CD4+ T cells to provide cellular growth factors and 'help'. CD4+ T cells recognize antigenic peptides in association with MHC class II molecules. Cells that express MHC class II peptides are typically professional antigen presenting cells such as dendritic cells or tissue-resident macrophages such as the Kupffer cells in the liver. Activation of malaria parasite-specific human CD4+ T cells requires presentation of parasite antigenic epitopes in association with human MHC class II molecules. If the CD4+ T cells were educated in a mouse thymus gland then there will be a skewing of the CD4+ T cell repertoire to recognize antigen in association with the mouse MHC II alleles present in that thymus. Education in human thymic tissue will be necessary if the T cells are to recognize predominantly antigen in association with human MHC molecules.

Engrafting human hepatocytes

Different models for hepatocyte engraftment have been described and are presented briefly here and in Table 1.

The first mouse strain that would accept human hepatocytes was the SCID/bg mouse with the urokinase type plasminogen activator gene (uPA) linked to an albumin promoter [30]. The immunodeficient mice have sub-acute liver failure and are readily transplanted with fresh or cryopreserved human hepatocytes at age 7-12 days via intrasplenic injection. Six to 8 weeks after transplantation with human hepatocytes, large islands of human liver tissue are produced within the mouse livers, creating a mouse with a human/mouse chimeric liver. When the human hepatocytes are successfully engrafted, 
the nodules of the human hepatocytes are visible and histologically integrated into the mouse liver. The rate of successful engraftment is $60-70 \%$ as determined by human serum albumin levels. This model has been used to study both viral hepatitis [24] and $P$. falciparum [30-32] in the absence of a human immune system. Others have recently attempted human lymphoid and myeloid cell engraftment in these mice[33].

In another model, mice were developed that are deficient in the gene for the tyrosine catabolic enzyme, fumarylacetoacetate hydrolase (Fah). These Fah-/- mice maintain their hepatocytes only in the presence of 2-(2-nitro-4-trifluoromethylbenzoyl)-1,3cyclohexanedione and lose them when the drug is withdrawn [34]. This gene deficiency was bred into immuno-deficient mice to create the FRG ${ }^{\mathrm{TM}} \mathrm{KO} / \mathrm{NOD}$ mouse. The mouse was recently reported to permit the development of $P$. falciparum sporozoites into exoerythrocytic forms in the liver [35]. Furthermore, when transplanted with human erythrocytes, the life cycle could be continued into the red cells [36]. It was recently shown that these mice can be given a human immune system [37] (Table 1).

An alternative model is the TK-NOG mouse in which mice express the herpes simplex virus thymidine kinase (HSVtk) transgenic construct containing the mouse albumin enhancer/promoter [38]. HSVtk mRNA is selectively expressed in the liver of NOG mice and they develop severe parenchymal liver damage after gancyclovir treatment [38]. Human liver cells are then injected intrasplenically (Table 1).

Caspase8-FK506 binding protein-albumin promoter transgenic mice offer a related approach in which the inducible death of hepatocytes occurs when FK 506 is dimerized. Balb/c RG mice were used to study hepatitis C infection in this study [39]. 
Mice have been engrafted with a human immune system (Table 1). Protection against infection was not assessed.

A different approach, recently described, is to inject human fetal hepatoblasts into immuoodeficient mice (NSG) which have been pre-treated with an anti-Fas antibody which induces murine hepatocyte apoptosis via a Caspase-3-deependent pathway (Bility MT et al., PLoS Pathogens e1004032, 2014). In this study, the mice also carried the human HLA-A2 transgene and received human stem cells, giving rise to functional human T cells (Table 1).

Engrafting human red cells or precursors

A powerful indicator of liver stage immunity from pre-erythrocytic malaria vaccines is the absence of blood stage parasites emerging from the liver. To measure blood stage $P$. falciparum infection in humanized mice, it is necessary to engraft mice with human erythrocytes (RBCs). When developing this model, the first strategy involved repeated injection with mature bovine erythrocytes into immune-compromised mice. Engraftment of human erythrocytes in mice was less consistent and occurred at lower levels of chimerism but such mice could sustain a P. falciparum infection [40-44].

The model was progressively improved with increasingly immune-deficient murine hosts [45-47]. These mice can sustain higher parasitemia with P. falciparum [48] and infection with multiple standard strains of $P$. falciparum without the need for speciesspecific parasite adaptation [49]. A recent advance in murine models of blood stage $P$. falciparum infection is the RBC-supplemented, immune cell-optimized humanized 
(RICH) mouse [50]. The need for repeated intra-peritoneal or intra-venous injections of uninfected human erythrocytes remains a limitation of this model.

The next anticipated major advance in humanized mouse models of blood stage malaria will be the xenotransplantation of human stem cells capable of recapitulating the entire cycle of erythrocyte development within the mouse. Recently, human hematopoietic stem cells along with human erythrocytes and plasmids encoding human erythropoietin and IL-3 were injected into recipient mice [51]. Significant erythrocyte chimerism ( 25\%) was observed, as well as a large fraction ( 40\%) of human PBMCs (peripheral blood mononuclear cells). In a further study with another immune-compromised strain that received human stem cells, HLADR4.RagKO.IL2R $\gamma c K O . N O D$ (DRAG) mice developed human erythrocytes and reticulocytes that represented approximately $0.2 \%$ of the red cell pool and could sustain an infection with P. falciparum following mosquito bite [52]. Low-level human hematocrit was due to poor differentiation of stem cells toward the erythrocytic lineage [52]. Further optimization of erythrocyte development in the murine host is needed to add more value to this new mouse model of $P$. falciparum blood stages.

Overcoming graft versus host disease and graft versus graft disease

Allogeneic haematopoietic stem cell transplantation in humans is sometimes complicated by the development of graft versus host disease (GVHD) mediated by donor immune cells responding to host antigens. In this disease the engrafted 
immune cells recognize the host tissues as ‘foreign' and the immune cells can cause significant tissue injury and death. Tissues typically affected by GVHD include the skin, the gut and the liver, but other tissues can be affected as well. Both innate and adaptive immune mechanisms are implicated in human GVHD. A similar disease can occur in mice transplanted with human immune cells; this is referred to as 'xenogeneic GVHD' in which human T cells that develop in the mouse respond to murine tissue as foreign antigens. HLL mice can be screened for GVHD by measuring a drop in serum human albumin, a rise in liver enzymes in the blood and clinical manifestations (skin changes, hair loss). Human PBMCs can engraft at high levels in NSG mice and produce a robust xeno-GVHD response [53-55]. XenoGVHD in this model appears to be largely mediated by donor T cell recognition of murine MHC molecules $[53,56]$. Of interest, investigators did not report significant levels of GVHD in a humanized mouse model of influenza using a mouse strain deficient in murine MHC class 1 (beta-2 microglobulin $(\beta-2 m)$ knockout) [57]. $\beta-2 m$ is a small peptide that stabilizes the expression of MHC class I molecules. Without expression of MHC class I molecules, the human immune cells will not recognize nor respond to the mouse cells.

Relative to PBMC and mature T-cells, cord blood CD34+ stem cells are associated with reduced levels of lethal GVHD when injected into NSG mice [58]. However, in the 'BLT' model where donor stem cells originate from fetal liver, late onset GVHD was reported with similarities to chronic GVHD in humans. 'BLT' refers to immunodeficient mice that have been reconstituted with human hematopoietic stem cells, and fragments of human fetal liver and thymus under the kidney capsule (Figure 2A) (Wege, AK et al, Current Topics Microbiol Immunol 324:149, 2008). However, 
further manipulations of BLT mice can reduce the levels of GVHD, such as the deletion of CD47. Reduced levels of GVHD were observed in R,G,CD47/--BLT mice compared to NSG-BLT mice [59].

Secondary lymphoid tissue

Secondary lymphoid tissue and B cells do not develop normally in many HIS or HLL mice. The reasons for this were recently reviewed [28] and relate in part to deficiencies in human cytokines necessary for lymphoid development. There is evidence that malaria sporozoites in the draining lymph nodes of skin can activate $\mathrm{T}$ cells and eliminate infected hepatocytes [60]. Thus, secondary lymphoid tissue may be critical to the development of optimal malaria-specific immune responses in HLL mice. It was recently shown that C57 BL6 Rag-/-, IL2R/-/-, CD47-/- BLT mice developed lymph nodes and spleens with follicles and normal B cells which underwent class switching, although antibody levels were significantly lower than in humans [59]. These mice, which require the implantation of human thymic tissue, have not been used to study malaria. HLL mice with secondary lymphoid tissue could also be ideal to study many malaria vaccines that rely on the production of antibodies.

\section{Perspective on the optimal HLL mouse for malaria vaccine research}

With the above considerations, we suggest that appropriate mouse models can be constructed for the study of liver stage immunity and vaccine development in HLL 
mice. With the goal to reconstitute the mouse with a population of functional $\mathrm{T}$ cells that can be stimulated by an experimental vaccine and which will then expand in number and recognize a malaria parasite-infected human hepatocyte population, consideration must be given to: (i) MHC matching between the CD34+ stem cells which will ultimately develop into mature $\mathrm{T}$ cell populations, the thymus where the $\mathrm{T}$ cells are educated, and the hepatocytes; and (ii) the frequency of human T cells that are present in the mouse that can be activated by malaria antigen. These two issues are related.

If the frequency of precursors is too low then it will be more difficult to activate sufficient cells to eliminate malaria-infected liver cells. In non-humanized mice, a large but defined number of $\mathrm{T}$ cells is required to effect immunity to the liver stages of malaria [61]. If $\mathrm{T}$ cells are educated in a mouse thymus then the number of cells that recognize foreign antigenic peptides in association with human MHC will be reduced. Although it is not known how many specific T cells will develop in any particular immunized HLL mouse, it seems reasonable to assume that education of T cells in a human thymus or a mouse thymus expressing human HLA antigens would be ideal. BLT mice offer this possibility but such mice would also need to express uPA or have a deletion of Fah (or use a different hepatocyte suicide strategy) for efficient engraftment with human hepatocytes. Figure 2A outlines a possible structure. However, there are major technical challenges and ethical issues with using human fetal tissue that limit the widespread suitability of this model.

Alternative models include transgenic mice that express human HLA antigens in their thymus (e.g. [52]) or are infected with a virus expressing human HLA genes [62] 
(Figure 2B). The T cells will develop from progenitors and be 'educated' in a HLAhumanized mouse thymus resulting in the repertoire being skewed favourably to recognize foreign antigens (e.g. malaria antigens) in association with human HLA. In these models it is also necessary that the thymus gland and the hepatocytes share the same human MHC class I antigen. This is not difficult to arrange if common HLA alleles are chosen, such as A2. GVHD is not a limiting factor with these mice as the T cells mature in a mouse where peripheral tissues (such as skin, kidney, pancreas, etc) and thymic cells express the same (murine) MHC molecules.

A third approach is: (i) begin with immunodeficient mice (e.g. NRG mice) stably engrafted with human CD34+ stem cells to generate antigen presenting cells; (ii) transfuse these mice with HLA-matched mature T cells from the same donor (e.g. from cord blood); (iii) use human hepatocytes that share at least one class I MHC molecule with these cells which will enable them to present malaria antigen to effector cytotoxic T-cells (Figure 2C). Breeding of the recipient mice to be deficient in $\beta-2 \mathrm{~m}$ mice will likely prevent GVHD. As mentioned above, $\beta-2 \mathrm{~m}$ is a molecule necessary to stabilize MHC class I molecules. Thus, the only cells that would possess $\beta-2 \mathrm{~m}$ in $\beta-2 \mathrm{~m}-/-$ mice and be recognized by $\mathrm{T}$ cells would be the donor cells of human origin. Mature human T cells have been transfused into $\beta-2 \mathrm{~m}-/-$ mice and GVHD was not reported [57]. An advantage of this model is that the mature CD4+ T cells are also educated in a thymus expressing human MHC antigens that are found on the antigen presenting cells (APCs) providing help for CD8+ T cells to expand [63]. This mouse may represent an ideal and relatively simple model for studying the immune response to malaria where human $\mathrm{T}$ cell responses could be tested against liver stage antigens and where vaccine efficacy could be monitored by measuring human red cell 
infection. This model could also be used to test immune responses to and efficacy of blood stage vaccines where $\mathrm{T}$ cell responses are critical [22]. Furthermore, the model could be adapted to ask whether T cells from vaccinated humans could kill malaria parasite-infected hepatocytes.

\section{Concluding remarks}

In this article, we summarized data relevant to the construction of mice humanized with both a human lymphoid system and hepatocytes suitable for liver stage malaria vaccine research. A current major impediment to progress in this field is the expense of early phase human studies and the difficulties with analyzing relevant immune responses that are occurring primarily in the liver. The HLL mouse offers a relatively simple alternative. While there are still a number of obstacles to be addressed, significant developments occurred over the last 5 years to enable the construction of various models of HLL mice. It is our view that the last two models described in Figure 2 represent the best current alternatives for further investigation.

While this review focused on the liver, a major tissue site for malaria parasites, similar issues are relevant to engraftment of other human tissues along with a nascent human immune system. Molecular, surgical and clinical tools are now available to readily produce various humanized mice that can be used for pre-clinical evaluation of novel vaccine approaches and to optimize delivery strategies. 
Figure Legends

Figure 1. Life cycle of malaria parasites in the human host. (Reproduced from [64].

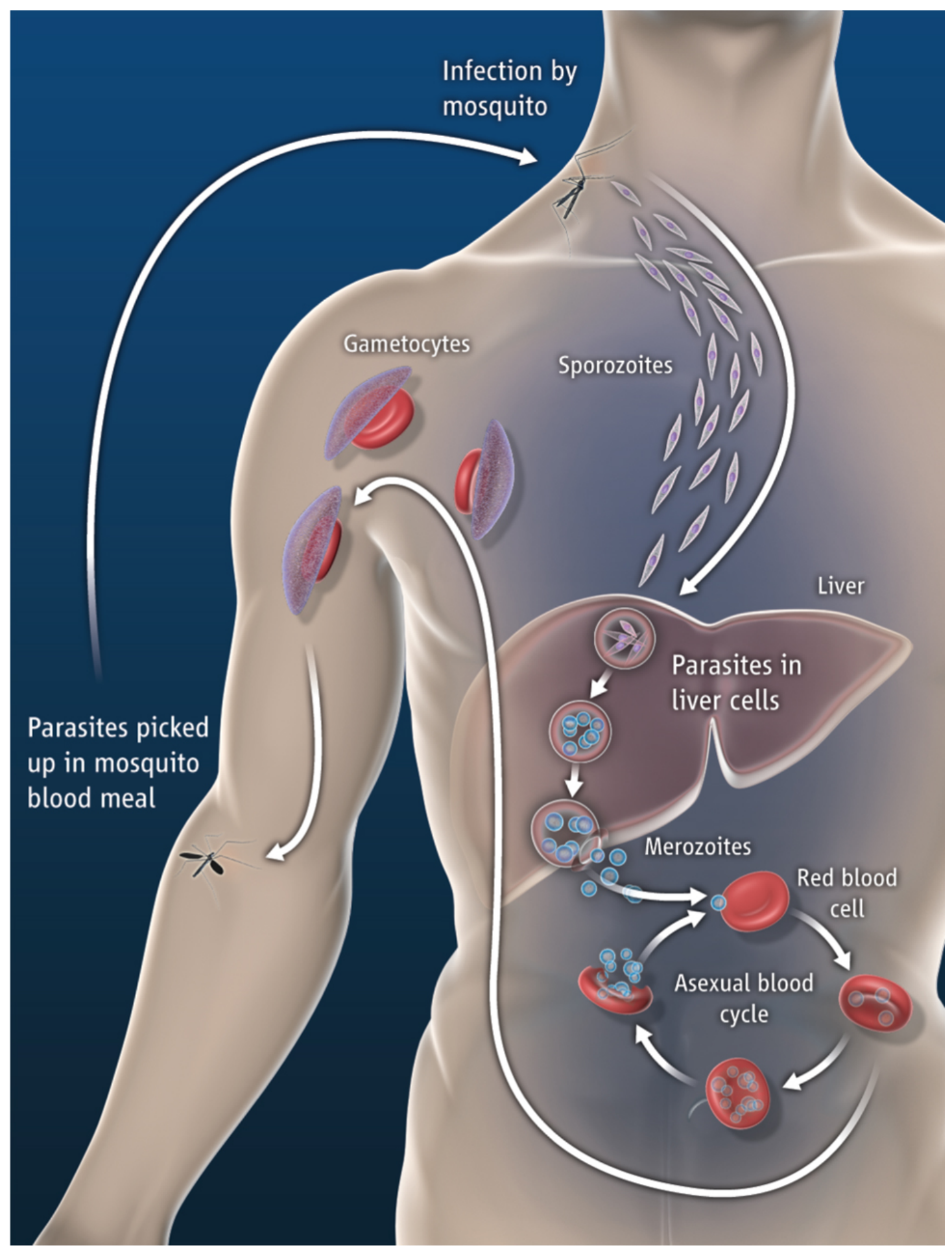


Figure 2. Models for generating a humanized leukocyte-liver (HLL) mouse. (A) The 'BLT' mouse (mice containing human stem cells, liver, and thymic grafts). This immune-deficient mouse is reconstituted with human hematopoietic stem cells (CD34+), and fragments of human fetal liver and thymus implanted under the kidney capsule. Antigen presenting cells (APCs) would derive from the human stem cells. Hepatocytes would need to express humanized major histocompatibility complex (MHC) I molecules (e.g. HLA-A2) to be recognized by humanized CD8+ T cells. Dendritic cells (DCs) and other APCs present antigen to CD4+ T cells in association with MHC II (e.g. HLA-DR4). To engraft human hepatocytes it would be necessary for these mice to express $u P A$, be deficient in Fah or use some other mouse hepatocyte suicide strategy. (B) Progenitor T cells and myeloid stem cells (APC progenitors) develop from human CD34+ (donor) stem cells. T cells develop in a thymus that is engineered to express human MHC I (e.g. HLA-A2) and MHC II (e.g. HLA-DR4). APCs (such as DCs or Kupffer cells) will express humanized MHC II and present antigenic epitopes to CD4+ T cells that were educated to recognize antigen in the context of human MHC II expressed in the thymus. (C) In this model, the mouse is engrafted with mature human T cells (CD4+ and CD8+). APCs will come from CD34+ stem cells that will be autologous to the donor T cells and allow recognition by the CD4+ T cells. In this model, it is necessary to use $\beta 2 \mathrm{~m}-/-$ recipient mice to prevent GVHD (graft versus host disease). Mice without $\beta 2 \mathrm{~m}$ will not express mouse MHC I molecules and will not be susceptible to GVHD. 


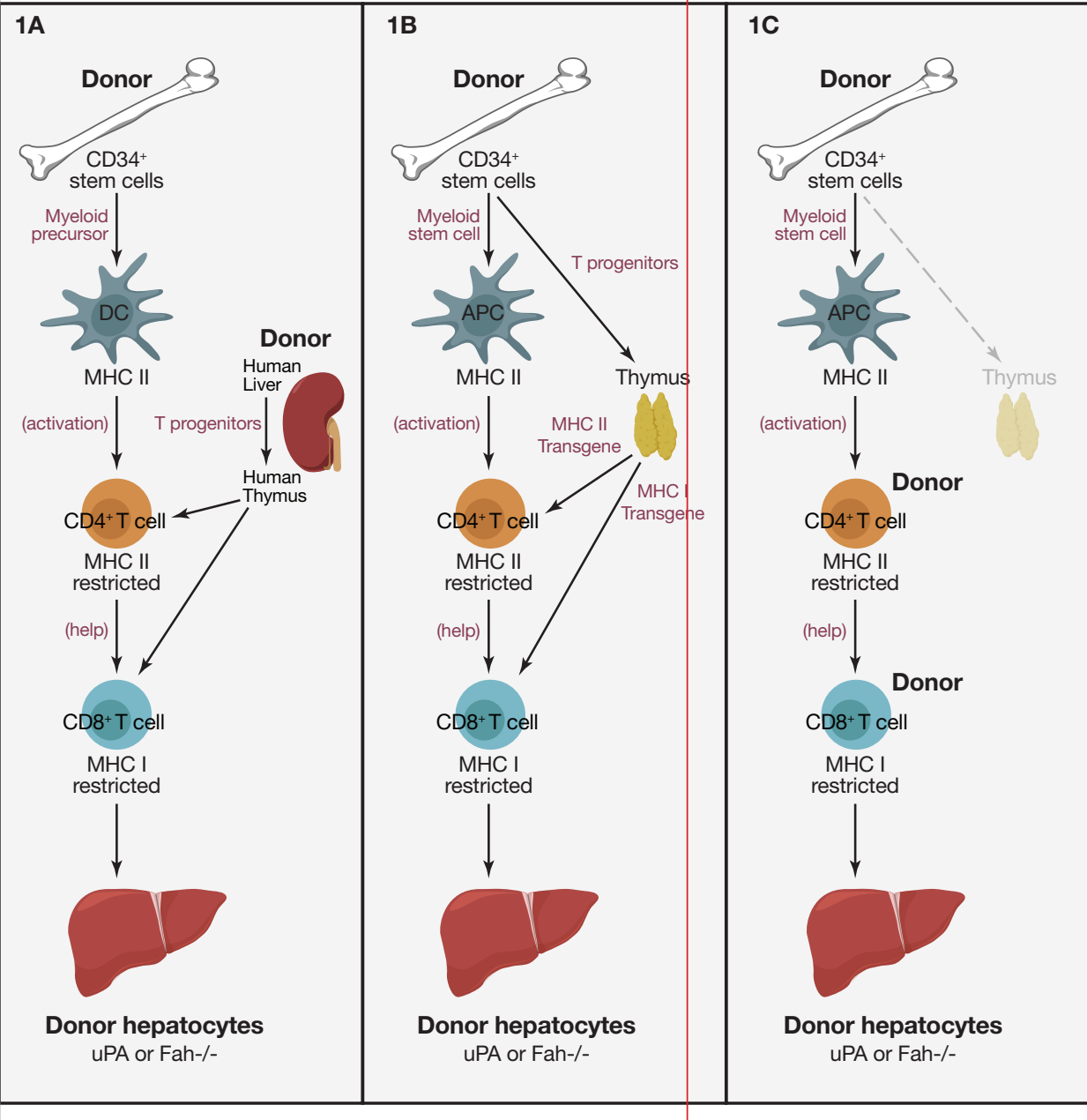




\section{Acknowledgements}

We thank Jahanara Rajwani for critically reviewing the manuscript. We thank Troy

Baldwin and D. Lorne Tyrrell for useful discussions. We acknowledge NHMRC (Australia), Alberta Innovates - Health Solutions, and the Women \& Children's Health Research Institute for grant support.

\section{Glossary}

Allogeneic - A mouse with a different genetic background.

APC - Antigen presenting cells (e.g. dendritic cells, Kupffer cells).

Autologous - Cells from the same animal.

$\beta 2 \mathrm{~m}$ - beta-2 microglobulin. An MHC Class I associated polypeptide that stabilizes the MHC molecule.

CD34 - Marker for pluripotent stem cells that can give rise to lymphoid and myeloid progenitors. These cells are also CD45 $\mathrm{dim}$.

CD47 - A trans-membrane protein ubiquitously expressed on human cells and, amongst other function, interacts with SIRP $\alpha$ on myeloid cells to inhibit phagocytosis.

Chimerism - Containing cells from two different individuals (here to mean mouse and human). 
Engraftment - Transplantation of foreign tissue.

Epitopes - Antigenic determinants from a protein or other molecule.

Fah-/- - These mice are deficient in fumarylacetoacetate hydrolase leading to tyropsineamia and death of hepatocytes. Mie can be protected with the drug 2-(2nitro-4-trifluoromethylbenzoyl)-1,3-cyclohexanedione (NTBC) and hepatocyte death will occur when the drug is withdrawn.

GVHD - Graft versus host disease.

Haematopoietic stem cells - Cells that give rise to all the other blood cells.

MHC I - Class I histocompatibility antigen expressed on all nucleated cells.

MHC II - Class II histocompatibility antigens expressed on professional antigen present cells.

NOD - The Non Obese Diabetic mouse whose SIRP $\alpha$ gene more closely resembles the human SIRP $\alpha$ gene, thus reducing phagocytosis of transplanted xenogeneic stem cells by myeloid cells.

NOG, NSG - Forms of NOD/SCID IL2R $\gamma-/-$ mice. 
Rag-/- - Recombination activating gene-deficient mice that cannot arrange immunoglobulin and $\mathrm{T}$ cell receptor genes. These mice are deficient in $\mathrm{T}$ and $\mathrm{B}$ cell function.

SCID - These mice are deficient in a DNA repair enzyme (Prkdc) preventing recombination in humoral and cellular immune response genes. They lack T and B cells and have similarities with the Rag-/- mice.

Secondary lymphoid tissue - Usually meant to indicate spleen and lymph nodes.

SIRP $\alpha$ - Signal regulatory protein alpha. It interacts with CD47 to negatively control phagocytosis.

uPA - Mice bearing the urokinase type plasminogen activator gene linked to an albumin promoter. These mice suffer from subacute liver disease and require transplanted normal hepatocytes for survival.

VDJ recombination - Somatic recombination of Ig or T cell receptor genes during lymphocyte development to generate diversity in the repertoire.

Xenotransplantation - Transplantation between two different species (here referring to human into mouse). 


\section{References}

1 Murray, C.J., et al. (2012) Global malaria mortality between 1980 and 2010: a systematic analysis. Lancet 379, 413-431

2 Schwartz, L., et al. (2012) A review of malaria vaccine clinical projects based on the WHO rainbow table. Malaria journal 11, 11

3 RTS,S Clinical Trials Partnership (2014) Efficacy and safety of the RTS,S/AS01 malaria vaccine during 18 months after vaccination: a phase 3 randomized, controlled trial in children and young infants at 11 African sites. PLoS medicine 11, e1001685 4 Hill, A.V. (2011) Vaccines against malaria. Philosophical transactions of the Royal Society of London. Series B, Biological sciences 366, 2806-2814

5 Ewer, K.J., et al. (2013) Protective CD8+ T-cell immunity to human malaria induced by chimpanzee adenovirus-MVA immunisation. Nature communications 4, 2836

6 Hoffman, S.L., et al. (2002) Protection of humans against malaria by immunization with radiation-attenuated Plasmodium falciparum sporozoites. The Journal of infectious diseases 185, 1155-1164

7 Seder, R.A., et al. (2013) Protection against malaria by intravenous immunization with a nonreplicating sporozoite vaccine. Science 341, 1359-1365

8 Epstein, J.E., et al. (2011) Live attenuated malaria vaccine designed to protect through hepatic CD8(+) T cell immunity. Science 334, 475-480

9 Nussenzweig, R.S., et al. (1967) Protective immunity produced by the injection of x-irradiated sporozoites of Plasmodium berghei. Nature 216, 160-162

10 Clyde, D.F., et al. (1973) Immunization of man against sporozite-induced falciparum malaria. The American journal of the medical sciences 266, 169-177 
11 Schofield, L., et al. (1987) Gamma interferon, CD8+ T cells and antibodies required for immunity to malaria sporozoites. Nature 330, 664-666

12 Weiss, W.R., et al. (1988) CD8+ T cells (cytotoxic/suppressors) are required for protection in mice immunized with malaria sporozoites. Proceedings of the National Academy of Sciences of the United States of America 85, 573-576

13 Corradin, G. and Levitskaya, J. (2014) Priming of CD8(+) T cell responses to liver stage malaria parasite antigens. Frontiers in immunology 5, 527

14 Tse, S.W., et al. (2011) Induction and maintenance of protective CD8+ T cells against malaria liver stages: implications for vaccine development. Memorias do Instituto Oswaldo Cruz 106 Suppl 1, 172-178

15 Cockburn, I.A., et al. (2013) In vivo imaging of CD8+ T cell-mediated elimination of malaria liver stages. Proceedings of the National Academy of Sciences of the United States of America 110, 9090-9095

16 Brehm, M.A., et al. (2010) Humanized mouse models to study human diseases. Current opinion in endocrinology, diabetes, and obesity 17, 120-125

17 Legrand, N., et al. (2009) Humanized mice for modeling human infectious disease: challenges, progress, and outlook. Cell host \& microbe 6, 5-9

18 Ploemen, I.H., et al. (2009) Visualisation and quantitative analysis of the rodent malaria liver stage by real time imaging. PloS one 4, e7881 19 Purcell, L.A., et al. (2008) Chemical attenuation of Plasmodium berghei sporozoites induces sterile immunity in mice. Infection and immunity 76, 1193-1199 20 Mueller, A.K., et al. (2005) Genetically modified Plasmodium parasites as a protective experimental malaria vaccine. Nature 433, 164-167 21 Bijker, E.M., et al. (2013) Protection against malaria after immunization by chloroquine prophylaxis and sporozoites is mediated by preerythrocytic immunity. 
Proceedings of the National Academy of Sciences of the United States of America $110,7862-7867$

22 Good, M.F., et al. (2013) Cross-species malaria immunity induced by chemically attenuated parasites. The Journal of clinical investigation

23 Mosier, D.E., et al. (1988) Transfer of a functional human immune system to mice with severe combined immunodeficiency. Nature 335, 256-259

24 Mercer, D.F., et al. (2001) Hepatitis C virus replication in mice with chimeric human livers. Nature medicine 7, 927-933

25 Yamauchi, T., et al. (2013) Polymorphic Sirpa is the genetic determinant for NOD-based mouse lines to achieve efficient human cell engraftment. Blood 121, $1316-1325$

26 Takenaka, K., et al. (2007) Polymorphism in Sirpa modulates engraftment of human hematopoietic stem cells. Nature immunology 8, 1313-1323

27 Kaushansky, A., et al. (2014) Of men in mice: the success and promise of humanized mouse models for human malaria parasite infections. Cellular microbiology 16, 602-611

28 Brehm, M.A., et al. (2014) Generation of improved humanized mouse models for human infectious diseases. Journal of immunological methods 410, 3-17 29 Klein, L., et al. (2014) Positive and negative selection of the T cell repertoire: what thymocytes see (and don't see). Nature reviews. Immunology 14, 377-391 30 Sacci, J.B., Jr., et al. (2006) Plasmodium falciparum infection and exoerythrocytic development in mice with chimeric human livers. International journal for parasitology 36, 353-360 
31 VanBuskirk, K.M., et al. (2009) Preerythrocytic, live-attenuated Plasmodium falciparum vaccine candidates by design. Proceedings of the National Academy of Sciences of the United States of America 106, 13004-13009 32 Morosan, S., et al. (2006) Liver-stage development of Plasmodium falciparum, in a humanized mouse model. The Journal of infectious diseases 193, 996-1004 33 Gutti, T.L., et al. (2014) Human hepatocytes and hematolymphoid dual reconstitution in treosulfan-conditioned uPA-NOG mice. The American journal of pathology 184, 101-109

34 Azuma, H., et al. (2007) Robust expansion of human hepatocytes in Fah-/-/Rag2-//Il2rg-/- mice. Nature biotechnology 25, 903-910

35 Vaughan, A.M., et al. (2012) Complete Plasmodium falciparum liver-stage development in liver-chimeric mice. The Journal of clinical investigation 122, 36183628

36 Vaughan, A.M., et al. (2012) Development of humanized mouse models to study human malaria parasite infection. Future microbiology 7, 657-665

37 Wilson, E.M., et al. (2014) Extensive double humanization of both liver and hematopoiesis in FRGN mice. Stem cell research 13, 404-412

38 Hasegawa, M., et al. (2011) The reconstituted 'humanized liver' in TK-NOG mice is mature and functional. Biochemical and biophysical research communications 405, $405-410$

39 Washburn, M.L., et al. (2011) A humanized mouse model to study hepatitis C virus infection, immune response, and liver disease. Gastroenterology 140, 13341344 
40 Tsuji, M., et al. (1995) Establishment of a SCID mouse model having circulating human red blood cells and a possible growth of Plasmodium falciparum in the mouse. Vaccine 13, 1389-1392

41 Moore, J.M., et al. (1995) Maintenance of the human malarial parasite, Plasmodium falciparum, in scid mice and transmission of gametocytes to mosquitoes. The Journal of experimental medicine 181, 2265-2270

42 Badell, E., et al. (1995) Human Plasmodium liver stages in SCID mice: a feasible model? Parasitology today 11, 169-171

43 Badell, E., et al. (2000) Human malaria in immunocompromised mice: an in vivo model to study defense mechanisms against Plasmodium falciparum. The Journal of experimental medicine 192, 1653-1660

44 Moreno, A., et al. (2001) Human malaria in immunocompromised mice: new in vivo model for chemotherapy studies. Antimicrobial agents and chemotherapy 45, $1847-1853$

45 Moreno Sabater, A., et al. (2005) Experimental infection of immunomodulated NOD/LtSz-SCID mice as a new model for Plasmodium falciparum erythrocytic stages. Parasitology research 95, 97-105

46 Rochford, R., et al. (2013) Humanized mouse model of glucose 6-phosphate dehydrogenase deficiency for in vivo assessment of hemolytic toxicity. Proceedings of the National Academy of Sciences of the United States of America 110, 1748617491

47 Angulo-Barturen, I., et al. (2008) A murine model of falciparum-malaria by in vivo selection of competent strains in non-myelodepleted mice engrafted with human erythrocytes. PloS one 3, e2252 
48 Jimenez-Diaz, M.B., et al. (2009) Improved murine model of malaria using Plasmodium falciparum competent strains and non-myelodepleted NOD-scid IL2Rgammanull mice engrafted with human erythrocytes. Antimicrobial agents and chemotherapy 53, 4533-4536

49 Arnold, L., et al. (2011) Further improvements of the P. falciparum humanized mouse model. PloS one 6, e18045

50 Chen, Q., et al. (2014) Human natural killer cells control Plasmodium falciparum infection by eliminating infected red blood cells. Proceedings of the National Academy of Sciences of the United States of America 111, 1479-1484 51 Chen, Q., et al. (2009) Expression of human cytokines dramatically improves reconstitution of specific human-blood lineage cells in humanized mice. Proceedings of the National Academy of Sciences of the United States of America 106, 2178321788

52 Wijayalath, W., et al. (2014) Humanized HLA-

DR4.RagKO.IL2RgammacKO.NOD (DRAG) mice sustain the complex vertebrate life cycle of Plasmodium falciparum malaria. Malaria journal 13, 386

53 King, M.A., et al. (2009) Human peripheral blood leucocyte non-obese diabeticsevere combined immunodeficiency interleukin-2 receptor gamma chain gene mouse model of xenogeneic graft-versus-host-like disease and the role of host major histocompatibility complex. Clinical and experimental immunology 157, 104-118 54 Abraham, S., et al. (2015) IL-10 exacerbates xenogeneic GVHD by inducing massive human T cell expansion. Clinical immunology 156, 58-64 55 Ali, N., et al. (2012) Xenogeneic graft-versus-host-disease in NOD-scid IL2Rgammanull mice display a T-effector memory phenotype. PloS one 7, e44219 
56 Covassin, L., et al. (2011) Human peripheral blood CD4 T cell-engrafted nonobese diabetic-scid IL2rgamma(null) H2-Ab1 (tm1Gru) Tg (human leucocyte antigen D-related 4) mice: a mouse model of human allogeneic graft-versus-host disease. Clinical and experimental immunology 166, 269-280

57 Yu, C.I., et al. (2008) Broad influenza-specific CD8+ T-cell responses in humanized mice vaccinated with influenza virus vaccines. Blood 112, 3671-3678 58 Gorin, N.C., et al. (2002) Increased risk of lethal graft-versus-host disease-like syndrome after transplantation into NOD/SCID mice of human mobilized peripheral blood stem cells, as compared to bone marrow or cord blood. Journal of hematotherapy \& stem cell research 11, 277-292

59 Lavender, K.J., et al. (2013) BLT-humanized C57BL/6 Rag2-/-gammac-/-CD47-/mice are resistant to GVHD and develop B- and T-cell immunity to HIV infection. Blood 122, 4013-4020

60 Chakravarty, S., et al. (2007) CD8+ T lymphocytes protective against malaria liver stages are primed in skin-draining lymph nodes. Nature medicine 13, 1035-1041 61 Schmidt, N.W., et al. (2008) Memory CD8 T cell responses exceeding a large but definable threshold provide long-term immunity to malaria. Proceedings of the National Academy of Sciences of the United States of America 105, 14017-14022 62 Huang, J., et al. (2014) An AAV vector-mediated gene delivery approach facilitates reconstitution of functional human CD8+ T cells in mice. PloS one 9, e88205

63 Janssen, E.M., et al. (2003) CD4+ T cells are required for secondary expansion and memory in CD8+ T lymphocytes. Nature 421, 852-856

64 Good, M.F. (2013) Immunology. Pasteur approach to a malaria vaccine may take the lead. Science 341, 1352-1353 